\title{
Mediators of allergic rhinitis: optimization of RNA isolation, reverse transcription, and $\mathrm{QPCR}$ protocols
}

\author{
Caroline Conway ${ }^{1}$, Jenny Thiele $2^{2^{*}}$, Mena Soliman ${ }^{1}$, Anne Ellis ${ }^{1,2,3}$ \\ From Canadian Society of Allergy and Clinical Immunology Annual Scientific Meeting 2014 \\ Ottawa, ON, Canada. 23-26 October 2014
}

\section{Background}

Optimizing methods for the study of allergic rhinitis (AR), especially when using samples likely containing small amounts of material for analysis, ensures the integrity of results that may potentially enhance the understanding of AR disease mechanisms. In order to conduct future mRNA expression analysis, examining the differential expression of AR mediators such as IL33, TSLPR, HPGDS, and CRTH2 at baseline and 6 hours following Nasal Allergen Challenge (NAC) in allergic individuals, this study aims to optimize the RNA isolation, reverse transcription (RT), and qPCR protocols used for the study of nasal mucosal samples.

\section{Methods}

Several RNA isolation and RT kits were evaluated using nasal scrapings from healthy individuals, similar to those collected from allergic participants. These kits were evaluated based on the yield and purity of RNA and cDNA, assessed using spectrophotometry, qPCR amplification, and gel electrophoresis. Reference gene analysis using cDNA isolated from allergic participants was conducted using qPCR and the statistical software GenEx (MultID). Primer design and evaluation of primers for the targets of interest-IL33, TSLPR, HPGDS, and CRTH2-was also pursued.

\section{Results}

RNA isolation and RT kit optimization determined that the Life Technologies-Qiagen (LT-Q) kit combination produced cDNA with maximal purity and qPCR efficiency compared with the other kit combinations evaluated. Reference gene analysis demonstrated that expression of ubiquitin $C$ (UBC) showed limited variability among the differing conditions (time point and study) of nasal sample collection. Primer evaluation yielded inconsistent results.

\section{Conclusions}

Future processing of nasal scraping samples should use the optimal LT-Q kit combination. Following successful primer evaluation, the expression levels of the targets of interest in the allergic nasal mucosal cDNA samples at both baseline and $6 \mathrm{~h}$ post-NAC will be conducted via the optimized $\mathrm{qPCR}$ reaction, using $\mathrm{UBC}$ as a reference gene.

\section{Authors' details}

'Department of Biomedical and Molecular Sciences, Queen's University, Kingston, Ontario, K7L 3N6, Canada. 'Department of Medicine, Queen's University, Kingston, Ontario, K7L 3N6, Canada. ${ }^{3}$ Division of Allergy \& Immunology, Kingston General Hospital, Kingston, Ontario, K7L 2V7, Canada.

Published: 18 December 2014

doi:10.1186/1710-1492-10-S2-A64

Cite this article as: Conway et al.: Mediators of allergic rhinitis: optimization of RNA isolation, reverse transcription, and qPCR protocols. Allergy, Asthma and Clinical Immunology 2014 10(Suppl 2):A64.

\footnotetext{
* Correspondence: jenny.thiele@queensu.ca

Department of Medicine, Queen's University, Kingston, Ontario, K7L 3N6,

Canada

Full list of author information is available at the end of the article
} 